\title{
Liver injury assessment by vetscan vs 2 analyzer and most frequently used alt/gtp reagent
}

\begin{abstract}
Background and aim: Liver injury is estimated by serum alanine aminotransferases (ALT) levels in experimental animal models. Laboratories use various techniques to measure ALT levels including assay reagents and chemistry analyzers. VetScan VS2 (VS2) is widely used in veterinary clinics and research laboratories for highly reproducible, convenient and effective testing. Alternatively, ALT liquid reagent is used by laboratories to estimate liver injury in animal studies. The aim of this study was to perform comparative analyses of data obtained from these two assays in two different animal models.
\end{abstract}

Methods: In this study, we used two different mouse models and compared the ALT levels measured using VetScan VS2 chemistry analyzer and ALT liquid reagent. Immunohistochemical analysis of hepatic tissue was also performed to document liver pathology. The first model is a high fat diet feeding model that results in a mild hepatic steatosis (fat accumulation in the liver) without elevation of ALT levels. For a severe liver injury model, we employed a hepatotoxin-induced liver injury model (carbon tetrachloride, $\mathrm{CCl}_{4}$ ), which leads to the development of hepatic fibrosis and very high ALT levels.

Results: VetScan VS2 and ALT reagent gave different values of ALT for all animal groups. However, linear regression analysis showed a significantly high association between ALT levels obtained by VS2 and ALT liquid reagent in a high-fat feeding model with no liver injury. For the $\mathrm{CCl}_{4}$ induced liver injury model, serum dilution (5 and 10 times) was performed to obtain accurate results with ALT reagent. ALT levels acquired from both techniques showed a close association. Interestingly, this correlation was closer when serum was diluted 5 fold.

Conclusion: This study demonstrates that both methods give similar results when evaluating liver injury in animal studies. However, the serum dilution factor is critical for severe liver injury assessment when using ALT reagent and requires some optimization. In this regard, VetScan VS2 is easier to use and gives comparable results.

Keywords: ALT, $\mathrm{CCl}_{4}$, Liver injury, VetScan VS2, stable liquid reagent, biochemical markers
Volume 4 Issue 4 - 2016

\author{
Vatsalya Vatsalya, ${ }^{1,3}$ Diana Avila, ${ }^{2}$ Jane \\ C Frimodig, ${ }^{4}$ Shirish S Barve, ${ }^{1,2}$ Craig J \\ McClain, ${ }^{1,2,3}$ Leila Gobejishvili ${ }^{1,2}$ \\ 'Department of Medicine, University of Louisville School of \\ Medicine, USA \\ ${ }^{2}$ Department of Pharmacology and Toxicology, University of \\ Louisville School of Medicine, USA \\ ${ }^{3}$ Robley Rex Veteran's Medical Center, USA \\ ${ }^{4}$ Department of Surgery, University of Louisville School of \\ Medicine, USA
}

Correspondence: Leila Gobejishvili, Department of Medicine, University of Louisville School of Medicine, 505 S. Hancock St. CTR Room 5I5, Louisville KY 40202, USA, Tel 502-852-036I, Fax 502-852-8927, Email IOgobe0I@louisville.edu

Received: January 26, 2016 | Published: April |3, 2016
Abbreviations: ALT, alanine amino transferases; $\mathrm{CCl}_{4}$, carbon tetrachloride; H\&E: hematoxylin and eosin; LDC, lieber-de carli; VS2: vetScan version2

\section{Introduction}

Liver injury is estimated by serum alanine aminotransferases (ALT) levels in experimental animal models. Laboratories employ various techniques to measure ALT levels. Several studies have evaluated liver injury using standard, conventional laboratory reagent assays, e.g. ALT (GPT) Stable Liquid Reagent. ${ }^{1-3}$ However, an increasing number of laboratories use chemistry analyzers for evaluation of liver function. Hence, chemistry analyzers have increasingly become a necessary tool in most clinical settings, as well as in pre-clinical laboratories. ${ }^{4}$ Both chemistry analyzer and testing performed in a preclinical lab have revealed concerns for reliability at pre-analytical, analytical, and post-analytical stages of evaluations. ${ }^{5}$ Factors that could influence pre-clinical testing include quality of the samples, insufficient reagent and sample storage, environmental changes such as analysis in hot or humid environments, other metabolic conditions that might alter accuracy level, and untrained/uncertified personnel. ${ }^{6}$ In this respect, chemistry analyzers require the least manual involvement, have a simplified process yielding to fewer errors compared to other assays, and produce immediate results. One such chemistry analyzer, VetScan VS2 (VS2) (Abaxis-North America, Union City CA), has been employed for measuring biochemical markers, including liver injury markers in small mammals. However, ALT (GPT) Stable Liquid Reagent is still a widely used method due to its low cost. The Stable Liquid Reagent determines ALT levels using three enzymatic reactions. When ALT levels are high (e.g. severe liver injury), these reaction rates exceed $0.26 / \mathrm{min}$ and serum must be diluted in order to get accurate results. Dilution factors vary depending on liver injury and require time and optimization. Selection of the wrong dilution factor could lead to either underestimation or overestimation of liver injury based on measured ALT levels. In this regard, the VS2 analyzer can measure ALT up to $2000 \mathrm{U} / \mathrm{L}$ and there is no need to perform serum dilutions.

The goal of this study was to determine the accuracy of the results generated from VetScan VS2 analyzer compared to the standard ALT/GPT Stable Liquid Reagent. We used two animal models with differing degrees of liver injury. In the first model, we fed mice a high fat containing diet, which leads to mild hepatic steatosis (lipid accumulation in the liver), but no liver injury. For the second model, we selected a hepatotoxin, carbon tetrachloride $\left(\mathrm{CCl}_{4}\right)$, to induce liver injury, which leads to severe liver damage and hepatic fibrosis. ${ }^{7}$ 


\section{Materials and methods}

\section{Animals}

12 week old male C57B1/6 mice were obtained from the Jackson Laboratory (Bar Harbor, ME). All mice were housed in a pathogenfree, temperature-controlled animal facility with 12- hour light-dark cycles. All experiments were carried out according to the criteria outlined in the Guide for Care and Use of Laboratory Animals and with approval of the University of Louisville Institutional Animal Care and Use Committee.

\section{High fat feeding animal model}

The Lieber-De Carli liquid diet (Lieber-De Carli type, Bioserv, Frenchtown, NJ) containing $40 \%$ fat was used for feeding the mice (HFD) for 6 weeks $(\mathrm{n}=16)$.

\section{$\mathrm{CC}_{14}$ induced liver injury model}

Carbon tetrachloride and corn oil were purchased from Sigma Aldrich (St. Louis, MO). $\mathrm{CCl}_{4}$ was dissolved in corn oil and administered at $1 \mathrm{ml} / \mathrm{kg}$ body weight two times a week for four weeks. Corn oil was used as a vehicle control. At sacrifice, mice were anesthetized with intraperitoneal injection of Avertin, $80 \mathrm{mg} /$ $\mathrm{kg}$. Whole blood was collected and plasma was stored at $-80^{\circ} \mathrm{C}$ for further analysis.

\section{Histopathology}

Formalin-fixed, paraffin-embedded liver sections were stained with Hematoxylin and Eosin (H\&E) for evaluation of histological changes and Sirius Red staining to detect collagen fibers. Frozen tissue was used for Oil Red O staining for lipid detection.

\section{Plasma ALT levels}

Plasma levels of alanine amino transferase (ALT) were measured using two techniques. First, ALT was measured using ALT reagents per the manufacturer's instructions (Thermo Fisher Scientific, Middletown, VA). Additionally, ALT levels were determined using a chemistry analyzer, VetScan VS2 (Abaxis North America), using VetScan prep profile II rotor.

\section{Statistical analysis}

These two study paradigms were evaluated independently by linear regression, as within group design analysis, comparing the estimate of the variance across the ALT data for each study sample between the two analysis methods. Linear regression analysis was also conducted in the $\mathrm{CCl}_{4}$ paradigm analysis to compare the association of ALT levels between the VS2 analyzer and ALT reagent assay. Further, we looked within the $\mathrm{CCl}_{4}$ group only and compared two levels of dilution factor ( $5 \mathrm{X}$ and $10 \mathrm{X}$ ) in the $\mathrm{CCl}_{4}$ paradigm, and measured the correlation of these data with the results obtained from the VS2 analyzer (Figure 1A-1D). A one tail t-test was used to compare the means of the ALT levels generated by the VS2 analyzer in the $\mathrm{CCl}_{4}$ and vehicle control group. MS Office (Microsoft Corp, Redmond WA), and SPSS 22.0 (IBM, Chicago, IL) were used in the statistical analysis. Statistical significance was set at $\mathrm{p} \leq 0.05$. All data are represented as Mean \pm SD.

\section{Results and discussion}

In the high fat feeding mouse model, onset of hepatic steatosis was confirmed by examination of liver tissues using H\&E and Oil Red O staining which showed accumulation of fat droplets in the liver tissue
(Figure 2). In this group, ALT levels obtained from two different analyses (VetScan and liquid reagent) showed different values (Figure $3)$. However, linear regression analysis showed significant robust association between the two ALT results indicating that both methods were comparable in assessing liver function (Figure 3) demonstrating that the VS2 method is accurate and comparable to the liquid reagent method.

To produce severe liver injury, we chose the well characterized $\mathrm{CCl}_{4}$ induced liver injury model. In this model, a hepatotoxin, $\mathrm{CCl}_{4}$, causes hepatocyte toxicity and death leading to uncontrollable activation and differentiation of hepatic stellate cells (HSC). HSCs express their differentiation marker, alpha smooth muscle actin ( $\alpha$ SMA) and produce extracellular matrix (ECM) including collagen. ${ }^{8-10} \mathrm{CCl}_{4}$ administration for 4 weeks led to the development of fibrosis with significantly increased immune-histochemical staining for aSMA protein in the liver (Figure 4). Examination of collagen deposition by Sirius Red staining of collagen fibers of the livers also showed a significant collagen expression in $\mathrm{CCl}_{4}$ group (Figure 4). As expected, ALT levels were significantly higher in mice treated with $\mathrm{CCl}_{4}$ when compared to untreated mice (control group) (Figure 5). Importantly, ALT levels of one $\mathrm{CCl}_{4}$ treated mouse exceeded the detection limit for the VetScan rotor and showed a level $>2000 \mathrm{U} / \mathrm{L}$. When we measured ALT with liquid reagent, mice treated with $\mathrm{CCl}_{4}$ (severe injury) had a reaction rate for ALT enzyme exceeding $0.26 / \mathrm{min}$. Thus, we diluted plasma according to manufacturer's instructions and performed the assay with two different dilutions (5X and 10X). Notably, the ALT levels obtained with $10 \mathrm{X}$ dilution and $5 \mathrm{X}$ dilution did not show high association (adjusted $\mathrm{R}^{2}=0.644$ ) (Figure 1D). We found a significantly high degree of association between the ALT levels generated by the VS2 and liquid reagent assay, in both $\mathrm{CCl}_{4}$, and control groups (Figure 1). In the $\mathrm{CCl}_{4}$ treated mice, this correlation between the ALT levels was much stronger than in the control group only at the $5 \mathrm{X}$ dilution (Figure 1A \& 1C). ALT levels analyzed at 10X dilution did not show the same strong association with VS2 ALT results (adjusted $\mathrm{R}^{2}=$ 0.729) (Figure 1B).

Results of this study showed that there was a high association between the two methods when ALT levels were relatively low; however for high ALT levels, this association was dependent on the dilution factor. In conclusion, we demonstrated a close association between ALT levels generated by VS2 and liquid reagent assay using two different mouse models. Both methods showed comparable results when used to assess milder injury, and VetScan VS2 presented more reproducible results with severe liver injury model.

It is important to note, that ALT liquid reagent used in this study, is recommended for human serum use. However, this reagent has been extensively used in animal studies to assess liver injury. ${ }^{11-15}$ This assay involves a series of enzymatic reactions. ${ }^{16,17}$ and must be performed at $37{ }^{\circ} \mathrm{C}$ using non-hemolysed serum or plasma, since erythrocytes have much higher ALT. ${ }^{18}$ Similarly, VetScan rotors are designed for veterinary use to analyze canine blood but have been used in experimental murine models. ${ }^{19}$ We took all precautions while analyzing plasma samples, including performing the tests in non-hemolysed samples. Additionally, the aim of this study was to compare two different methods of ALT estimation in mouse models and demonstrate the limitations of each method. Our results show that ALT reagent should be used with caution when liver injury is severe. Optimization of the dilution of serum or plasma samples is critical to accurately estimate liver injury in order to avoid either under or overestimation of liver damage..$^{20}$ This becomes particularly relevant when the study involves the assessment of therapeutic intervention in modulating liver injury. 

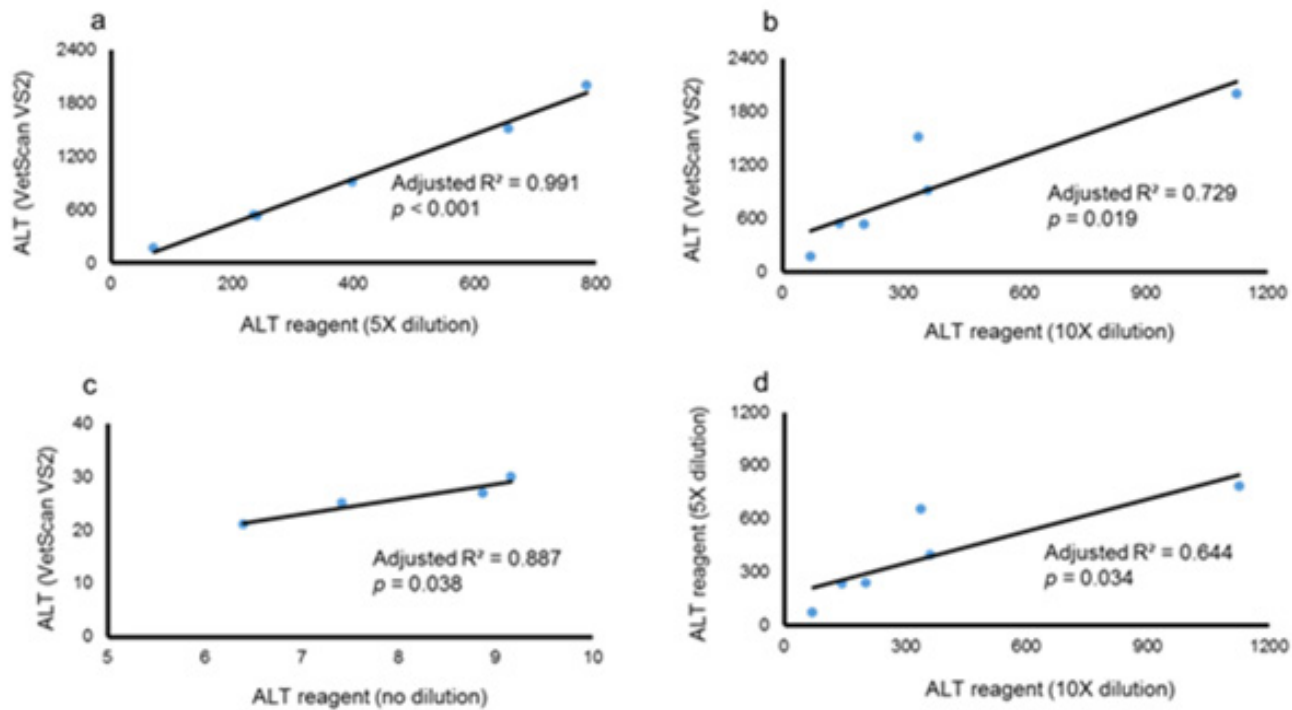

Figure Ia ALT levels compared using the VS2 results and $5 X$ dilution used in the ALT reagent assay. Unstandardized coefficient, $B=2.516 \pm 0.107 ;$ zero-order standardized $b=0.996$.

Figure Ib ALT levels comparing VS2 results and IOX dilution used in ALT reagent assay. Unstandardized coefficient, B $=1.589 \pm 0.418$; and zero-order standardized $\mathrm{b}=0.885$.

Figure Ic ALT levels compared using VS2 results and $5 X$ dilution used in ALT reagent assay in controls. Unstandardized coefficient, $B=2.809 \pm 0.566$; and zeroorder standardized $b=0.962$

Figure Id Association of ALT levels obtained using the ALT reagent method, $5 \mathrm{X}$ and I0X dilution factors. Unstandardized coefficient, $B=1.189 \pm 0.375 ;$ zeroorder standardized $b=0.846$.

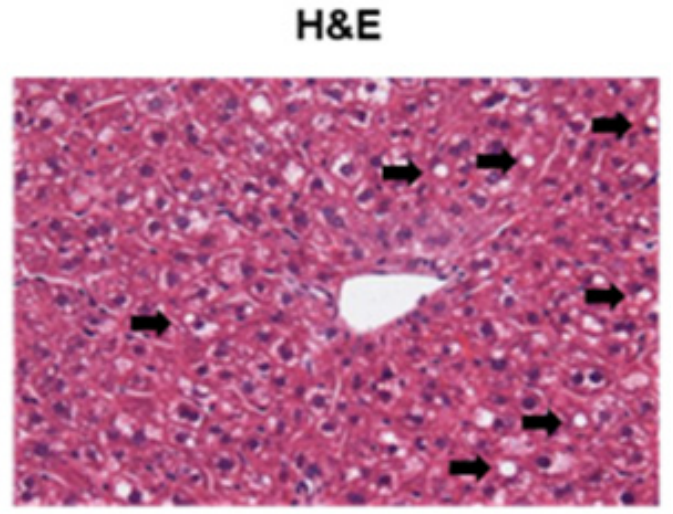

\section{Oil Red O}

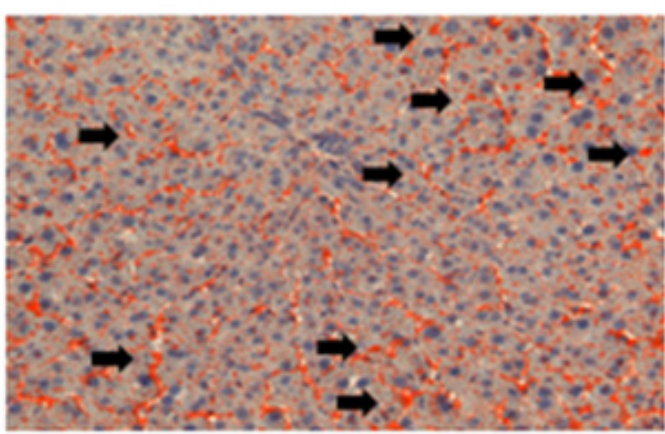

Figure 2 Hematoxylin-Eosin (H\&E) and Oil Red $O$ staining of liver tissue of mice on LDC diet (arrows indicate lipid droplets).

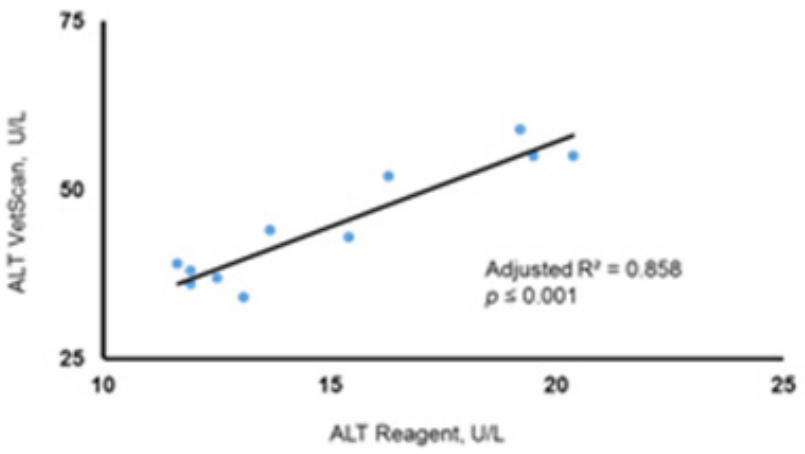

Figure 3 Association of the ALT levels obtained by VetScan VS2 and ALT reagent assay. There was a high degree of correlation between the two methods in assessing ALT in the LDC diet mice. Unstandardized coefficient, $B$ $=2.515 \pm 0.321$; zero-order standardized, $\mathrm{b}=0.934$.

\section{Acknowledgment}

We thank Marion McClain for editing the manuscript. This work was supported by a grant from National Institute on Alcohol Abuse and Alcoholism (NIAAA) R21AA022189 (LG).

\section{Contribution to publication}

LG, VV and JF designed the study project. DA, LG, and VV contributed in conducting the experiment. VV and LG contributed to analysis of the data and interpretations. VV, SSB and LG wrote the manuscript. SSB and CJM provided valuable scientific contribution to enhance the explanation of the results.

Citation: Vatsalya V, Avila D, Frimodig JC, et al. Liver injury assessment by vetscan vs2 analyzer and most frequently used alt/gtp reagent. Gastroenterol Hepatol Open Access. 20 I6;4(4):96-100. DOI: 10. I5406/ghoa.20I6.04.00 I07 
Control

\section{H\&E}

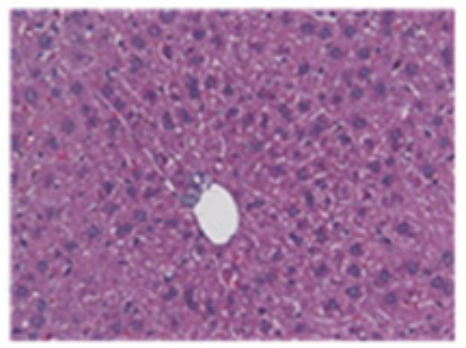

\section{Sirius Red}

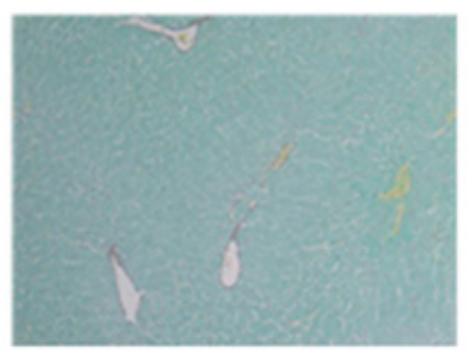

$\mathrm{CCl} 4$
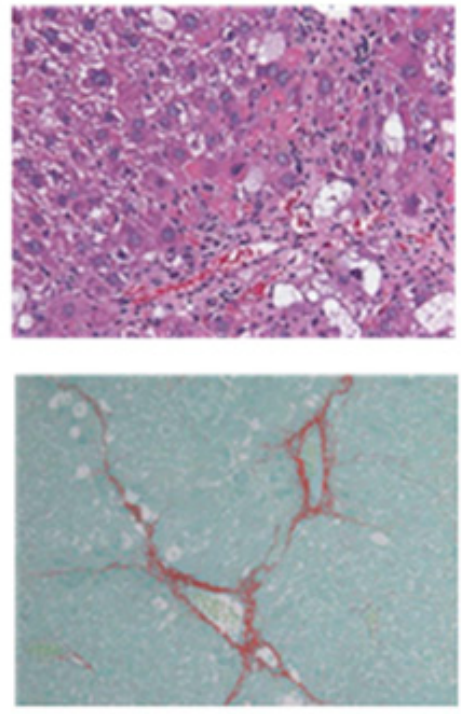

Figure $4 \mathrm{CCl} 4$ administration resulted in a significant liver damage and collagen deposition. (Top) H\&E staining; (Bottom) Sirius Red staining.

a

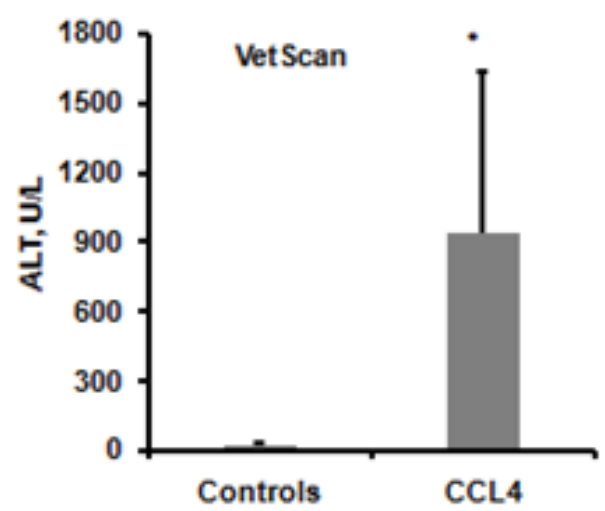

b

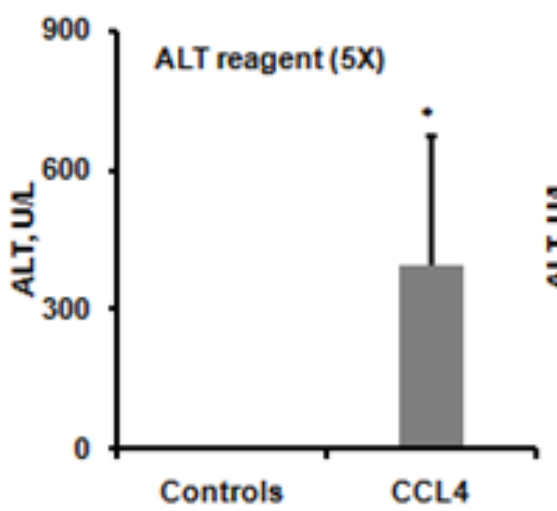

c

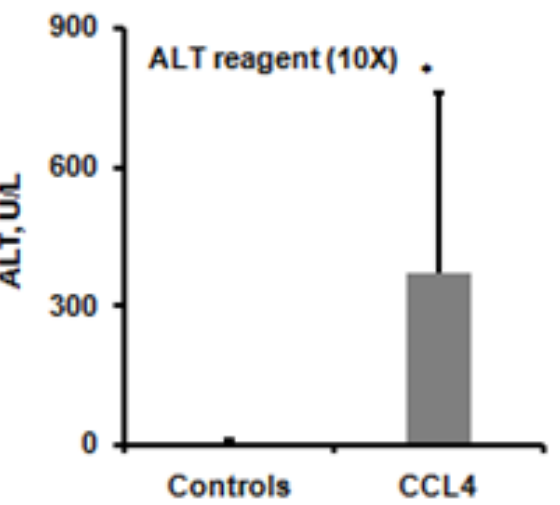

Figure 5 ALT levels in the control and $\mathrm{CCl} 4$ group. $\mathrm{CCl} 4$ group showed significant elevation in ALT levels compared to the controls,a. ALT Levels obtained by using VetScan, b. ALT levels obtained using ALT liquid reagent (5X), c. ALT levels obtained using ALT liquid reagent (I0X). P $<0.05$. Data are presented as Mean \pm SD.

\section{Funding}

None.

\section{References}

1. Czaja MJ, Xu J, Alt E. Prevention of carbon tetrachlorideinduced rat liver injury by soluble tumor necrosis factor receptor. Gastroenterology. 1995;108(6):1849-1854.

2. Gobejishvili L, Barve S, Breitkopf Heinlein K, et al. Rolipram attenuates bile duct ligation-induced liver injury in rats: a potential pathogenic role of PDE4. J Pharmacol Exp Ther. 2013;347(1):80-90.
3. Wang X, Yan S, Xu D, et al. Aggravated liver injury but attenuated inflammation in PTPRO-deficient mice following LPS/D-GaIN induced fulminant hepatitis. Cell Physiol Biochem. 2015;37(1):214224.

4. Price CP. Regular review: point of care testing. $B M J$. 2001;322(7297):1285-1288.

5. Nichols JH. Point of care testing. Clin Lab Med. 2007; 27(4):893-908.

6. Westgard JO, Klee GO. Quality assurance. In: Tietz (Ed.), Fundamentals of clinical chemistry. (3rd edn), WB Sanders, Philadelphia, PA, USA. 1987:238-253. 
7. Dong D, Xu L, Yin L, et al. Naringin prevents carbon tetrachlorideinduced acute liver injury in mice. J Funct Foods. 2015;12:179-191.

8. Jiao J, Friedman SL, Aloman C. Hepatic fibrosis. Curr Opin Gastroenterol. 2009;25(3):223-229.

9. Friedman SL. Mechanisms of hepatic fibrogenesis. Gastroenterology. 2008;134(6):1655-1669.

10. Kisseleva T, Brenner DA. Anti-fibrogenic strategies and the regression of fibrosis. Best Pract Res Clin Gastroenterol. 2011;25(2):305-317.

11. Gobejishvili L, Barve S, Breitkopf Heinlein K, et al. Rolipram attenuates bile duct ligation-induced liver injury in rats: a potential pathogenic role of PDE4. J Pharmacol Exp Ther. 2013;347(1):80-90.

12. Song M, Chen T, Prough RA, et al. Chronic alcohol consumption causes liver injury in high-fructose-fed male mice through enhanced hepatic inflammatory response. Alcohol Clin Exp Res. 2016;40(3):518 528.

13. Massey VL, Poole LG, Siow DL, et al. Chronic alcohol exposure enhances lipopolysaccharide-induced lung injury in mice: potential role of systemic tumor necrosis factor-alpha. Alcohol Clin Exp Res. 2015;39(10):1978-1988.
14. Kirpich I, Zhang J, Gobejishvili L, et al. Binge ethanol-induced HDAC3 down-regulates cpt $1 \alpha$ expression leading to hepatic steatosis and injury. Alcohol Clin Exp Res. 2013;37(11):1920-1929.

15. Wu KC, Liu JJ, Klaassen CD. Nrf2 activation prevents cadmiuminduced acute liver injury. Toxicol Appl Pharmacol.2012;263(1):14-20.

16. Bergmeyer HU, Scheibe P, Wahlefeld AW. Optimization of methods for aspartate aminotransferase and alanine aminotransferase. Clin chem. 1978;24(1):58-73.

17. Henry RJ, Chiamori M, Golub OJ, et al. Revised spectrophotometric methods for the determination of glutamate oxaloacetic transaminase, glutamic pyruvic transaminase and lactic acid dehydrogenase. $\mathrm{Am} \mathrm{J}$ Clin Pathol. 1960;34:381-398.

18. Burtis CA, Ashwood ER. Tietz textbook of Clinical Chemistry. (2nd edn). 1994.

19. Lee JH, Kim K, Jo YH, et al. Therapeutic hypothermia attenuates liver injury in polymicrobial sepsis model of rats via Akt survival pathway. $J$ Surg Res. 2013;181(1):114-120.

20. Fraser $\mathrm{CG}$, Fogarty Y. Interpreting laboratory results. $B M J$. 1989;298(6689):1659-1660. 